\title{
The Comparison Analysis of Opinions Towards the Concept of Consumer Health Informatics among Korean and American Health Informatics Academic Society Members
}

\author{
Jeongeun Kim \\ Graduate School of Health Science Yonsei University, Seoul Korea
}

\begin{abstract}
Objectives: Consumer Health Informatics (CHI) is an increasingly important research area within health informatics, however, it is as yet not a well recognized discipline. The purpose of this study was obtaining consensus on a definition and description of CHI among members of health informatics related society in Korea and the United States. Design \& Measurement: The CHI-WG "Survey of Definitions of CHI" was administered via the World Wide Web (www) in English and Korean. Results: Differences found between two groups were opinions on the definition of $\mathrm{CHI}$ and in recognition of importance for related disciplines. Conclusion: Visions and recommendations for the future of CHI are provided.
\end{abstract}

Keywords: Consumer Health Informatics, Internet

\section{Introduction}

A new era of health care is emerging. Increasingly educated consumers are demanding convenience in accessing health information, participation in decision-making about treatments, and choice in selection of health care, etc. Perhaps the most common health care consumer activity today is that of health-information seeking.

These consumers are bringing about changes in the health care industry, and in professionals behavior in interacting with consumers[1]. Increasingly, professionals and consumers engage in interactive health communication. 1 . Potential benefits of consumer online health-information seeking are; widespread access to health information, 2. interactivity, tailoring of information, potential to facilitate interpersonal interaction and social support, potential for 3 . anonymity.

By contrast, the potential harms and hazards of online 4. health-information are; inequitable access to relevant information, navigational difficulties, information overload, disorganization, searching difficulties, inaccessible or overly technical language, lack of user friendliness, lack of permanence, lack of peer review or regulation, inaccurate/misleading/dangerous information, lack of consumers' evaluation skills, risk-promoting messages abound, potential for online pathologies and maladaptive behaviors[2].

The Consumer Health Informatics Working Group (CHIWG) became an official IMIA Working Group in 2000. The CHIWG is concerned with electronic information related to health care available to the public (e.g. Internet, wireless, standalone electronic media). For its purposes, it defines Consumer Health Informatics(CHI) as "the use of modern computers and telecommunications to support consumers in obtaining information, analyzing unique health care needs and helping them make decisions about their own health" [3], in which the consumer interacts with the applications directly with or without the presence of health care professionals. The group's interests focus on, but are not limited to, world wide web sites that offer advice about healthy living, research findings, and recommendations on specific disease conditions, descriptions of products, medications, and self-care health programs available to the public. As described above, there are many questions concerned about $\mathrm{CHI}$ as it is in the emerging stage, and many research issues to be considered and studied. The purpose of this survey is to define the components of a description of $\mathrm{CHI}$ and to understand the relative importance of issues for future study.

\section{Purpose}

To define the components and types of $\mathrm{CHI}$ and issues to be addressed and their relative importance, arriving at a quantitative consensus.

\section{Objectives}

1. To identify important components of a description of CHI.

To identify the relative importance of specific issues for a research agenda in CHI.

To identify $\mathrm{CHI}$ research activities at member's institutions.

To compare the differences of opinions between Korean and American groups

\section{Methods}

Based on the purposes mentioned above, a questionnaire was developed by IMIA CHIWG members. The survey questions are: Indicate which of the following subjects should be included in a description of Consumer Health Informatics(CHI) activities., Rate how important each of the following areas of expertise are to development, 
implementation, and evaluation of Consumer Health Informatics interventions. (Rate each on a scale from: 1=Very unimportant, 2=Unimportant, $3=$ Neutral, 4=Important, 5=Very important), Indicate the single most important issue to be incorporated into future Consumer Health Informatics research., Are there any ongoing informatics activities (i.e., program development, research, education) at your institution that focus of Consumer Health Informatics? Would you recommend that Consumer Health Informatics be considered a separate discipline within Health Informatics?, etc. English version questionnaire was translated into Korean by a Korean nursing informatics scholar, and back translated by native and bilingual English speaker. Both English version and Korean version questionnaires were converted into web forms, and uploaded on the Korean professional survey conducting vendor's server to implement the online survey. By obtaining the email lists of Korean and American societies that are concerned with medical and health informatics (Korean Society of Medical Informatics and Capital Area Roundtable on Informatics in NursinG), survey participation soliciting emails were sent to the members. Data were collected from $14^{\text {th }}$ until $22^{\text {nd }}$ December 2002 for Korean members, and from $1^{\text {st }}$ until $8^{\text {th }}$ February 2003 for American members. The reasons of the time lag between two groups were programming and translating issues. Data were analyzed using SAS version 8.1 to identify means, standard deviations and statistical significance of differences between two groups. The Cronbach's Alpha was 0.67 for the reliability testing of the instruments.

\section{Results}

135 Koreans out of 566, and 64 Americans out of 767 were responding. There were statistical differences for item number $1,2,3,7$, and 8 . Item 1 Consumer health information - provision of patient education through technology, item 2 Patient access to their own electronic medical records, and item 7 Security/Confidentiality issues were agreed higher in Americans than Koreans. Item 3 Consumer health commerce - advertising for consumer goods, and item 8 Patient-health care provider communication and relationships issues were agreed higher in Koreans than Americans.

There were statistical differences in recognition of importance for computer science, health science, and health education science between 2 groups. Koreans evaluated computer science higher, whereas Americans evaluated health science and health education science.

The comparison of order of importance of research issues in $\mathrm{CHI}$ showed no statistical difference between 2 groups, even though it seems that Americans rated outcomes research such as impact of CHI on health outcomes, service utilization and etc very high .

The comparison of ongoing informatics activities showed no statistical difference between 2 groups.

The comparison of degree of agreement on the CHI as an independent discipline showed no statistical difference between 2 groups. Both groups agree modestly that $\mathrm{CHI}$ as an independent discipline.

There were statistical differences for comparison of percentage of working group participation, as Americans showed much higher participation rate for American Medical Informatics Association that is natural. Other participation rates showed no differences.

\section{Discussions}

In 1990, Shortliffe and Perrault wrote that for health professionals "it is increasingly difficult to practice modern medicine without information technologies" a statement that is more true than ever today[4]. However, these days there is an additional trend: it is also increasingly unlikely that health professionals will encounter patients who have not used information technology to influence their health knowledge, health behavior, perception of symptoms, and illness behavior. Health professionals should, therefore, not only understand consumer health applications butalso ensure that these applications are developed, applied, and evaluated properly.

There is another issue to be considered seriously other than consumer health information usage. Although the information society offers tremendous potential for reducing the knowledge gap between professionals and patients, it also brings a risk of a widening of the gap between those who have access to new technology and those who have been excluded[5]. Bridging this digital divide and bringing consumer health informatics to groups that have the greatest need will be particularly challenging. In the industrial age, the inverse care law described the idea that the availability of good medical care tends to vary inversely with the need for medical care in the population served[6]. In the information age, we face an analogous "inverse information law" that is, access to appropriate information is particularly difficult for those who need it most. The vicious circle of low education and low health literacy and low income, poor health, and the inaccessibility of information technology, can only be broken if the field is not left to market forces alone but if public health policy actively brings information technology to those who are underserved.

As such, there are many issues that should be researched to empower consumers of health related information. Several interesting results were found by this survey.

Firstly, it could be reasoned that the reason of low response rate of American members was that the e-mails containing some Korean characters or some meta-statements that made those e-mails be treated as some kind of spam mails and be deleted automatically by the receiving servers. Korean members' low response rate could be conjectured by the fact that some e-mails were returned because of unknown addresses. Conducting international survey using e-mails has some tricky aspect that researchers should be cautious. They need to pay attention to raise the response rate more than usual survey that is conducted in one country.

Americans seems to have more interests in provision of education, and accessibility at the same time 
security/confidentiality. Koreans have more interests in advertising online, as Korea's infrastructure for e-commerce[1] has grown up very rapidly to give them more convenience of[2] purchasing products. That could be the reason why Korean health informatics professionals think that consumer health commerce needs attention. The reason of "patient-health care provider communication and relationships" issue was[3] rated higher in Koreans than in Americans could be[4] conjectured that patient could not have enough time with their health care provider in Korean health care delivery system, and it is one of the main complaints of Korean[5] patients. Korean people think it is very important to have close relationships with others, but that could not be the case in patient-health care provider. It could be predicted and hoped that the technology and $\mathrm{CHI}$ discipline improve the relationships in the near future.

It seems that Koreans have more focus on technology,[6] whereas Americans have more focus on health discipline and health education. Using technology to empower patients as consumers is very brand new notion in Korea, and that makes health informatics professionals have more attention to technology out of computer science.

Other than above mentioned issues, most opinions about CHI showed no remarkable differences between two groups, as there should be some consensus about CHI's role and impact internationally. It would be interesting, however, to find some cultural differences on consumers' status and empowerment through the advancement and adoption of technology in healthcare arena between countries in the future researches.

\section{Conclusions}

Even though a little differences were found between Koreans and Americans, it could be concluded that $\mathrm{CHI}$ is considered as important and essential discipline that needs to be developed and interested in both countries. It is clear that by introducing and utilizing $\mathrm{CHI}$ research proactively will empower consumers of health information in this digital age regardless of the region in the world. The greatest contribution of $\mathrm{CHI}$ research to the healthcare sector may eventually be found in its attempts to systematize and codify consumers' needs, values, and preferences; in its research into how information is digested and is best presented to consumers; and in its research into how these variables influence outcome measures. Thus, current health informatics research may have greater implications for the practice of medicine than medical informatics ever did before. And also, empowerment of consumers of health related information using $\mathrm{CHI}$ researches should be more enlightened, activated and consolidated.

\section{Acknowledgement}

The author thanks Betty L. Chang, DNSc, FNP-C, FAAN, School of Nursing University of California, Los Angeles, USA for her great efforts for initiating and organizing this research and her thoughtful review of this manuscript.

\section{References}

Bopp, KD, Stud Health Technol Inform 2000;76:93-106

Cline, R.J.W. and Haynes, K.M., Consumer health information seeking on the Internet: the state of the art, Health Education Research, Vol. 16, No. 6, 671-692, December 2001

U.S. General Accounting Office, 1996, p.1.

Shortliffe EH, Perrault L. Medical informatics: computer applications in health care. Reading, MA: Addison-Wesley, 1990.

Irving L, Klegar-Levy K, Everette DW, Reynolds T, Lader W. Falling through the net: defining the digital divide. $A$ report on the telecommunications and information technology gap in America. Washington, DC: National Telecommunications and Information Administration, US Dept of Commerce, 1999.

Hart JT. The inverse care law. Lancet 1971; i: 405-412.

\section{Address for Correspondence}

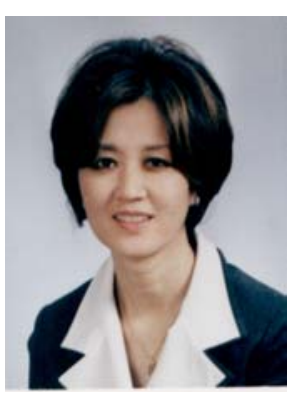

Jeongeun Kim is a research professor at Yonsei University Graduate School of Health Science, where she gives on-line lectures on Nursing Informatics, Systems Analysis and Design, etc. She graduated from and took her doctorate at Seoul National University, and finished post-doctoral fellowship at University of Utah College of Nursing for Nursing Informatics. Her major research interests are CAI/WBI, Consumer Health Informatics and Patient Safety. Interested readers may contact the author, via either jekim@yumc.yonsei.ac.kr or Graduate School of Health Science Yonsei University, 134 Shinchon-dong Seodaemungu Seoul, Korea 120-752 